DOI: 10.17533/udea.efyd.v33n1a09

URL DOI: http://doi.org/10.17533/udea.efyd.v33n1a09

\title{
NIVELES Y ESTADOS DE CAMBIO DE LA ACTIVIDAD FÍSICA EN UNA COMUNIDAD UNIVERSITARIA DE MEDELLÍN-COLOMBIA'
}

\author{
NÍVEIS E ESTADOS DE ALTERAÇÃO DA ATIVIDADE \\ FÍSICA EM UMA COMUNIDADE UNIVERSITÁRIA \\ DE MEDELLÍN-COLÔMBIA
}

\author{
PHYSICAL ACTIVITY LEVELS AND STAGES \\ OF CHANGE OF A UNIVERSITY COMMUNITY \\ IN MEDELLÍN-COLOMBIA
}

\section{Víctor Hugo Arboleda Serna}

Mag ster en Salud Colectiva por la Universidad de Antioquia

Profesor e Investigador del Grupo de Investigaci $n$ en Ciencias Aplicadas a la Actividad F sica y el Ejercicio (GRICAFDE) del Instituto de Educaci $n$ F sica de la Universidad de Antioquia (Colombia)

vihuas@gmail.com

Elkin Fernando Arango Vélez

Magister en Epidemiolog a $\mathrm{Cl}$ nica, Especialista en Medicina Deportiva.

Arboleda S., V. H; Arango V., E. F.; Feito, Y. (2014). Niveles y estados de cambio de la actividad $\mathrm{f}$ sica en una comunidad universitaria de medell n-colombia. Educaci $n$ F sica y Deporte, 33 (1), 153-173, Ene-Jul 2014

1 Este art culo es un producto asociado a la investigaci n "Niveles, estados de cambio, beneficios y barreras frente a la actividad $\underline{f \text { sica }}$ en la comunidad universitaria de Ciudadela Robledo, Universidad de Antioquia. Desarrollado con el apoyo del Grupo de Investigaci n GRICAFDE. 
Profesor e Investigador del Grupo de Investigaci n en Ciencias Aplicadas a la Actividad F sica y el Ejercicio (GRICAFDE) del Instituto de Educaci n F sica de la Universidad de Antioquia (Colombia)

elkinarango@yahoo.com

\section{Yuri Feito}

Ph.D, MPH, Ciencias del Deporte por la Universidad de Tennesse Profesor Asistente en el Departamento de Ciencias del Ejercicio y Administraci $\mathrm{n}$ del deporte en la Kennesaw State University (USA). yfeito@kennesaw.edu

\section{RESUMEN}

Este estudio se plante como objetivos determinar los niveles y los estados de cambio frente a la actividad $f$ sica en la comunidad universitaria del campus "Ciudadela de Robledo" de la Universidad de Antioquia, Medell n, Colombia. Participaron de manera voluntaria 92 estudiantes, 72 docentes y 45 empleados; utilizando un muestreo aleatorio simple para cada grupo. Se emple el Cuestionario Global de Actividad F sica (GPAQ) para identificar los niveles de actividad f sica, y el Sample Physical Activity Questionnaire para los estados de cambio; las encuestas fueron recolectadas durante el segundo semestre del a o 2012. Se manej el programa estad stico SPSS versi n 21 para el an lisis de los datos, utilizando $t$ cnicas de distribuci n de frecuencias para realizar el an lisis descriptivo; las variables cualitativas fueron analizadas de acuerdo al v nculo con la universidad y al sexo, utilizando la prueba de $\mathrm{X}^{2}$ para comparar las proporciones. Pudo identificarse que el $51,1 \%$ de los estudiantes, el $48,6 \%$ de los docentes y el 46,7\% de los empleados presentan niveles altos de AF. La mayor proporci n encontrada para los estados de cambio de acuerdo al $v$ nculo, la obtuvo el estado de mantenimiento, 47,2\% para los docentes, 28,9\% para los empleados y $26,1 \%$ para los estudiantes. Los resultados del presente estudio podr an servir como insumos para la creaci $n$ de estrategias que permitan mejorar los programas deportivos y de AF del campus universitario, y busquen incrementar los niveles de AF de la poblaci $n$.

PALABRAS CLAVE: Actividad f sica, estados de cambio, comunidad universitaria

\section{RESUMO}

Traçou-se como objetivo principal deste estudo determinar os n veis e os estados de alteraç o frente atividade $\mathrm{f}$ sica na comunidade universit ria do 
campus "Ciudadela de Robledo" da Universidade de Antioquia, Medell n, Col mbia. Participaram de maneira volunt ria 92 estudantes, 72 docentes e 45 empregados; utilizando uma amostragem aleat ria simples para cada grupo. Se empregou o Question rio Global de Atividade F sica (GPAQ) para identificar os n veis de atividade f sica, e o Sample Physical Activity Questionnaire para os estados de alteraç o; as enquetes foram coletadas durante o segundo semestre do ano de 2012. Empregou-se o programa estat stico SPSS vers o 21 para a an lise dos dados, utilizando t cnicas de distribuiç o de frequ ncias para realizar a an lise descritiva; as vari veis qualitativas foram analisadas de acordo com o v nculo com a universidade e com o sexo, utilizando a prova de $X^{2}$ para comparar as proporç es. $P$ de-se identificar que $51,1 \%$ dos estudantes, 48,6\% dos docentes e 46,7\% dos empregados apresentam n veis altos de AF. A maior proporç o encontrada para os estados de alteraç o de acordo com o v nculo foi obtida pelo estado de manutenç o, 47,2\% para os docentes, $28,9 \%$ para os empregados e $26,1 \%$ para os estudantes. Os resultados do presente estudo poderiam servir como insumos para a criaç o de estrat gias que permitam melhorar os programas esportivos e de AF do campus universit rio, e busquem incrementar os $n$ veis de AF da populaç $\mathrm{o}$.

PALAVRAS-CHAVE: Atividade $\mathrm{f}$ sica, estados de alteraç $\mathrm{o}$, comunidade universit ria

\section{ABSTRACT}

The main goal of this study was to determine the levels of physical activity and stages of change related to it in the "Ciudadela de Robledo" campus of the University of Antioquia, Medellin, Colombia. A total of 92 students, 72 faculty's members and 45 employees voluntarily participated in this study. The Global Physical Activity Questionnaire (GPAQ) was used to identify the physical activity levels and the Sample Physical Activity Questionnaire to determine the stages of change. The surveys were collected during the second semester of 2012. The data was handled and analyzed using SPSS version 21(Statistical program) applying frequency distribution techniques to develop the descriptive analysis; the qualitative variables were analyzed according to the university nexus and gender using the $\mathrm{X}^{2}$ test to compare the proportions. The results evinced the $51.1 \%$ of students, $48.6 \%$ of professors, and $46.7 \%$ of employees presented high PA levels. The biggest proportion for the stages of change according to the nexus was obtained by the maintenance stage $47.2 \%$ for professors, $28.9 \%$ for employees, and $26.1 \%$ for students. The results from this study could serve as a basis to create strategies that allow the improvement of sportive and PA programs inside the campus in order to increase the PA level among the campus population.

KEYWORDS: Physical activity, stages of change, college campus, questionnaires, descriptive 


\section{INTRODUCCIÓN}

Seg n cifras de la Encuesta Nacional de la Situaci n Nutricional en Colombia (ENSIN), el exceso de peso en la poblaci $n$ colombiana aument del 45,9\% en 2005 al 51,2\% en 2010, mostr ndose que esta situaci $\mathrm{n}$ es mayor en mujeres $(55,2 \%)$ que en hombres $(45,6 \%)$; as mismo, la mayor prevalencia se presenta en el rea urbana (52,5\%) superando el promedio nacional (ICBF et al., 2011)

Con respecto a la Actividad F sica (AF), solo uno de cada tres colombianos cumple con las recomendaciones semanales de 150 minutos de ejercicio f sico cardiovascular a intensidad moderada (US Department of Health and HumanServices, 2008); sin embargo, se observ un aumento de 3,4 puntos porcentuales en la prevalencia de cumplir con estas recomendaciones entre el ENSIN 2005 y el 2010 (ICBF et al., 2011). Los niveles recomendados de AF en Colombia, en concordancia con las recomendaciones internacionales, mostraron que la AF en el tiempo libre y caminar como medio de transporte, tienen una prevalencia del $53,5 \%$ en los adultos de 18 a 64 a os; para la ciudad de MedeII n y sus corregimientos (SSM, 2008), la prevalencia de baja AF es de $54,4 \%$; y a nivel mundial se encuentra que la inactividad f sica es de $31,1 \%$, siendo mayor para las mujeres, comparadas con los hombres, $33,9 \%$ y $27,9 \%$ respectivamente; para la regi $\mathrm{n}$ de las Am ricas es de 43,2\% (Hallal et al., 2012).

En este sentido, la inactividad $\mathrm{f}$ sica se constituye en el cuarto factor de riesgo $\mathrm{m}$ s importante de mortalidad en el mundo, supe$r$ ndolo la hipertensi $\mathrm{n}$ arterial, el consumo de tabaco, el exceso de glucosa en la sangre, el sobrepeso y la obesidad; de la misma manera, se evidencia que cada vez $\mathrm{m}$ s personas en el mundo son inactivas $f$ sicamente, lo cual repercute significativamente en la salud mundial y aumenta la prevalencia de enfermedades $\mathrm{Cr}$ nicas no trasmisibles (ECNT) (OMS, 2010). En esta direcci n, el bajo nivel de AF se relaciona tanto a nivel mundial como regio- 
nal como uno de los factores de riesgo asociados con los altos ndices de ECNT, convirti ndose en un asunto de gran inter $\mathrm{s}$ valorar la AF desde diversas variables y grupos poblacionales, entre los cuales se hallan las poblaciones universitarias.

En un estudio desarrollado con 500 estudiantes universitarios de la Universidad de las Flores, Argentina, se encontr que el $87 \%$ de los estudiantes en educaci $\mathrm{n}$ f sica cuentan con niveles de AF altos, comparados con los estudiantes de otras carreras; adicionalmente, se identific que esta relaci $n$ se invierte en las categor as de AF baja y moderada, para las cuales los niveles $\mathrm{m}$ s elevados los tienen los estudiantes de otras carreras diferentes a los de educaci $\mathrm{n}$ f sica (Farinola, 2011).

En una investigaci $\mathrm{n}$ realizada en la Universidad de Caldas, Colombia, se identificaron, entre otras variables, los niveles de AF de los empleados de la instituci $n$ y los estados de cambio de la $A F$, encontrando que el $51,3 \%$ de los empleados son suficientemente activos, el $37,2 \%$ insuficientemente activos y el $11,5 \%$ son muy activos; en relaci $\mathrm{n}$ con los estados de cambio, el $74,4 \%$ de los empleados est $n$ en la fase de contemplaci $n$ (Pe a, Gallo \& V squez, 2009).

Resultados de una investigaci $\mathrm{n}$ desarrollada con empleados y docentes de una universidad en la ciudad de Medell $n$, Colombia, indican que el $45,4 \%$ de los evaluados eran sedentarios, $33,7 \%$ eran activos y 20,9\% muy activos; el principal factor de riesgo fueron las dislipemias y los valores $\mathrm{m}$ s bajos de colesterol LDL y de depresi $\mathrm{n}$ correspondieron a las personas f sicamente activas o muy activas (Aguilar, Zapata, Giraldo, Tejada \& Vidales, 2008).

Ahora bien, la Universidad de Antioquia, en su estrategia Universidad saludable (UdeA, 2005) plantea, en la I nea de acci $n$ relacionada con ambientes saludables, la formulaci $n$, ejecuci $n$ y evaluaci $n$ de proyectos que promuevan una gesti $n$ ambiental y ecol gica en procura de espacios acad micos y laborales confortables; as mismo, expone en otra de sus I neas de acci $n$ 
el fomento de $\mathrm{h}$ bitos y estilos de vida saludable a trav $\mathrm{s}$ de las actividades $\mathrm{f}$ sicas y deportivas; en este sentido, y asumiendo la responsabilidad adquirida por la universidad, se hace necesario desarrollar investigaciones que permitan conocer los niveles de AF y los estados de cambio frente a la AF que tiene su poblaci n, puesto que no se han desarrollado hasta la fecha investigaciones que indaguen sobre esta problem tica. Con base en los resultados obtenidos en el presente estudio, se busca contribuir al desarrollo e implementaci $\mathrm{n}$ de programas de AF orientados de acuerdo con las caracter sticas particulares de su poblaci n, permitiendo ajustar y mejorar los programas de AF existentes y quiz s la creaci $n$ de nuevos programas que garanticen una mayor contribuci $\mathrm{n}$ a la salud y mayor adherencia a los mismos.

\section{Objetivo}

Determinar los niveles de AF e identificar los estados de cambio de la comunidad universitaria del campus "Ciudadela de Robledo" de la Universidad de Antioquia.

\section{MÉTODO}

El presente fue un estudio cuantitativo de car cter descriptivo, transversal.

\section{Población y muestra}

La poblaci $n$ total fue de 2806 personas entre estudiantes, docentes y empleados pertenecientes a la comunidad universitaria de la sede "Ciudadela Robledo" de la Universidad de AntioquiaColombia. Para la selecci $n$ de los participantes se utiliz un muestreo probabil stico de car cter aleatorio simple, empleando para el c Iculo del tama o de la muestra el programa EPIDAT versi $\mathrm{n} 4.0$, con un nivel de confianza del $95 \%$ y error $\mathrm{m}$ ximo del $5 \%$; con una probabilidad de ocurrencia del evento (bajo ni- 
vel de actividad f sica) de 0.47; estratificado de acuerdo al v nculo con la universidad, lo que dio como resultado una muestra total de 592 personas, discriminada as : 327 estudiantes, 198 docentes y 67 empleados, a los cuales se les trat de contactar empleando diversas estrategias (afiches, correos electr nicos, llamadas telef nicas, a trav s de personas conocidas, visita a su oficina o puesto de trabajo); se obtuvo una frecuencia de respuesta global del $35,3 \%$, para los estudiantes del $28,1 \%$, docentes del $36,3 \%$ y empleados del $67,1 \%$. La informaci $\mathrm{n}$ se recolect durante el segundo semestre del a o 2012.

\section{Instrumentos}

Las caracter sticas sociodemogr ficas fueron identificadas a tra$\mathrm{v}$ s de un cuestionario que incluy preguntas como sexo, edad, $\checkmark$ nculo con la universidad (docente, empleado estudiante), estrato socioecon mico ( 1 al 6), y estado civil. Los docentes son los profesores nombrados o contratados para desarrollar actividades de investigaci $n$, docencia, extensi $n$ y administraci $n$ acad mica, quienes pueden ser vinculados (de tiempo completo o de medio tiempo), o contratados (ocasionales, visitantes, ad hon rem o c tedra); los tres primeros podr n ser de tiempo completo o de medio tiempo por $\mathrm{t}$ rmino inferior a un a o; los de c tedra son contratados por horas (UdeA, 1996). El estrato socioecon mico se entiende como una clasificaci $n$ en estratos de los inmuebles residenciales que deben recibir servicios $p$ blicos; se realiza principalmente para cobrar de manera diferencial por estratos los servicios $\mathrm{p}$ blicos domiciliarios, permitiendo asignar subsidios y cobrar contribuciones; quienes tienen $\mathrm{m} \mathrm{s}$ capacidad econ mica pagan $\mathrm{m} s$ por los servicios $\mathrm{p}$ blicos $\mathrm{y}$ contribuyen para que los estratos bajos puedan pagar sus tarifas (DANE, sf).

Para determinar los niveles de AF se emple el Cuestionario Global de Actividad F sica (GPAQ)(OMS, 2007) en su versi n en espa ol, el cual ha sido utilizado y validado en diversas 
investigaciones (Abu-Omar \& R tten, 2008; Bauman, Nelson, Pratt, Matsudo \& Schoeppe, 2006; Bull, Maslin, \& Armstrong, 2009), dentro de las cuales se encuentra evidencia con poblaciones universitarias (Farinola, 2011). Este instrumento permite establecer los niveles de AF de acuerdo con una escala (bajo, medio y alto) basada en las recomendaciones internacionales de promoci $n$ de la AF para la salud. En el nivel alto se clasifican aquellas personas que realizan AF de intensidad vigorosa al menos $3 \mathrm{~d}$ as por semana, alcanzando un $\mathrm{m}$ nimo de $1500 \mathrm{MET} / \mathrm{min} / \mathrm{semana}$, o $7 \mathrm{~d}$ as de AF entre moderada y vigorosa, logrando un m nimo de $3000 \mathrm{METs} / \mathrm{min} / \mathrm{semana}$. Para el nivel de AF moderado es necesario que las personas cumplan con alguno de los siguientes criterios: a) 3 o m s d as de AF a intensidad vigorosa con una duraci $\mathrm{n}$ m nima de 20 minutos por $\mathrm{d}$ a; b) $5 \mathrm{o} \mathrm{m} \mathrm{s} \mathrm{d}$ as de AF a intensidad moderada con una duraci $\mathrm{n}$ m nima de 30 minutos por $\mathrm{d} \mathrm{a}$; $\mathrm{c}) 5 \mathrm{o} \mathrm{m} \mathrm{s} \mathrm{d}$ as de AF entre moderada y vigorosa que alcance al menos $600 \mathrm{MET} /$ min/semana. Por Itimo, en el nivel bajo de AF se clasifican aquellas personas que no cumplen con cualquiera de los criterios mencionados.

La identificaci n de los estados de cambio de la AF se llev a cabo a trav s del Sample Physical Activity Questionnaire (The Centers for Disease Control and Prevention, 2010) el cual se compone de cinco preguntas que permiten clasificar a la persona en uno de los estados de cambio frente a la AF: precontemplaci $n$ (personas que no realizan AF en el tiempo libre ni tienen la intenci $\mathrm{n}$ de hacerla en los pr ximos seis meses), contemplaci $n$ (personas que no realizan AF en el tiempo libre pero tienen la intenci $\mathrm{n}$ de hacerla en los pr ximos 6 meses), preparaci $n$ (personas que no realizan AF en el tiempo libre pero tienen la intenci $\mathrm{n}$ de hacerla en los pr ximos $30 \mathrm{~d}$ as), acci $n$ (personas que realizan $\mathrm{AF}$ regular en el tiempo libre desde hace por lo menos 6 meses) y mantenimiento (personas que realizan AF regular en el tiempo libre desde hace $\mathrm{m} s$ de 6 meses). 


\section{Criterios de selección}

Fueron incluidas todas las personas vinculadas con la Universidad de Antioquia durante el primer semestre de 2012 como estudiante, docente o empleado, en los programas acad micos que se ofrecen en el campus Ciudadela de Robledo. Se excluy a quienes no aceptaron participar o se encontraban en comisi $n$ (administrativa o de estudios) o incapacidad laboral.

\section{Control de sesgos}

Para controlar los sesgos en la selecci n de los participantes, se emple un muestreo probabil stico de car cter aleatorio simple para cada uno de los v nculos con la universidad (docente, empleado, estudiante). Los sesgos de informaci n se controlaron con la aplicaci $\mathrm{n}$ del mismo instrumento para la recolecci $\mathrm{n}$ de datos, que se someti a una prueba piloto para corregir las preguntas de dif cil comprensi n. Se utilizaron herramientas estandarizadas internacionalmente para la recolecci $\mathrm{n}$ de la informaci n (GPAQ y Sample Physical Activity Questionnaire); aquellas personas que no aceptaron participar o no se lograron localizar, se reemplazaron de manera aleatoria a partir de la base de datos existente, de acuerdo a su v nculo con la universidad.

\section{Análisis Estadístico}

Para el an lisis de los datos se utiliz el programa estad stico SPSS versi n 21 y Excel 2010 para Windows. Las variables cuantitativas se resumieron mediante medias y desviaciones est ndar. Las variables cualitativas, como los niveles de AF (alto, moderado y bajo) y estados de cambio (precontemplaci n, contemplaci n, preparaci $n$, acci $n$ y mantenimiento) fueron analizadas de acuerdo al v nculo con la universidad y al sexo, y sus resultados presentados en frecuencias y proporciones, utilizando la prueba de $X^{2}$ para comparar las proporciones, estableciendo diferencias estad sticamente significativas con valores de $(p<0,05)$. 


\section{RESULTADOS}

Características sociodemográficas de la muestra

Con base en las 209 personas participantes del estudio, se encuentra que el $53,6 \%$ son mujeres y el $46,4 \%$ hombres, con una edad media de 33,9 DE 11,7 a os para los hombres y 33,4 a os DE 11,2 para las mujeres; de ellos el $44 \%$ tienen el $v$ nculo de estudiantes, $34,4 \%$ son docentes y el $21,5 \%$ son empleados. Otras caracter sticas sociodemogr ficas se muestran en la Tabla 1.

\begin{tabular}{|c|c|c|c|}
\hline \multicolumn{2}{|l|}{ Variables } & \multirow{2}{*}{$\begin{array}{c}\text { Frecuencia } \\
97\end{array}$} & \multirow{2}{*}{$\begin{array}{c}\text { Porcentaje } \\
46,4 \%\end{array}$} \\
\hline & Hombre & & \\
\hline sexo & Mujer & 112 & $53,6 \%$ \\
\hline \multirow{2}{*}{ Edad promedio } & Hombre 33,9 años DE 11,7 & 97 & $46,4 \%$ \\
\hline & Mujer 33,4 años DE 11,2 & 112 & $53,6 \%$ \\
\hline \multirow{3}{*}{$\begin{array}{l}\text { Vínculo con la } \\
\text { Universidad }\end{array}$} & Docente & 72 & $34,4 \%$ \\
\hline & Empleado & 45 & $21,5 \%$ \\
\hline & Estudiante & 92 & $44 \%$ \\
\hline \multirow{6}{*}{$\begin{array}{l}\text { Estrato } \\
\text { socioeconómico }\end{array}$} & Estrato 1 & 7 & $3,3 \%$ \\
\hline & Estrato 2 & 35 & $16,7 \%$ \\
\hline & Estrato 3 & 96 & $45,9 \%$ \\
\hline & Estrato 4 & 54 & $25,8 \%$ \\
\hline & Estrato 5 & 16 & $7,7 \%$ \\
\hline & Estrato 6 & 1 & $0,5 \%$ \\
\hline \multirow{5}{*}{ Estado civil } & Soltero & 129 & $61,7 \%$ \\
\hline & Casado & 56 & $26,8 \%$ \\
\hline & Unión libre & 15 & $7,2 \%$ \\
\hline & Viudo & 2 & $1 \%$ \\
\hline & Separado/Divorciado & 7 & $3,3 \%$ \\
\hline
\end{tabular}

Tabla1. Características sociodemográficas 
Poblaci n del campus universitario Ciudadela Robledo vinculada con el Instituto Universitario de Educaci n F sica, la Facultad de Ciencias Agrarias y la Escuela de Nutrici n y Diet tica.

\section{Niveles de Actividad Física}

Con relaci $\mathrm{n}$ al nivel de actividad $\mathrm{f}$ sica, se logr determinar que el $51,1 \%$ de los estudiantes tienen un nivel de AF alto, seguido por los docentes $48,6 \%$ y los empleados $46,7 \%$. Igualmente, los estudiantes reportan mayor nivel de AF moderada (26,1\%) comparados con los docentes y empleados $(18,1 \%$ y $20 \%$, respectivamente). Con respecto a la clasificaci n de AF baja, se encuentra que el $33,3 \%$ de los docentes y los empleados, al igual que el 22,8\% de los estudiantes, no cumplen los m nimos de AF para mejorar su salud. En esta muestra, no se identificaron diferencias estad sticamente significativas entre los diferentes $v$ nculos con la universidad ( $p=0,321)$. Ver Tabla 2.

\begin{tabular}{|c|c|c|c|c|c|c|}
\hline \multirow{2}{*}{\multicolumn{2}{|c|}{$\begin{array}{l}\text { Vinculo con la } \\
\text { Universidad }\end{array}$}} & \multicolumn{3}{|c|}{ Nivel de Actividad Física } & \multirow{3}{*}{$\begin{array}{c}\text { Total } \\
72\end{array}$} & \multirow{3}{*}{$\begin{array}{c}\text { P de } \\
\text { tendencia }\end{array}$} \\
\hline & & \multirow{2}{*}{$\begin{array}{c}\text { Alto } \\
35\end{array}$} & \multirow{2}{*}{\begin{tabular}{|c|} 
Moderado \\
13
\end{tabular}} & \multirow{2}{*}{$\frac{\text { Bajo }}{24}$} & & \\
\hline Docente & Frecuencia & & & & & \\
\hline & Porcentaje & $48,6 \%$ & $18,10 \%$ & $33,30 \%$ & $100 \%$ & \multirow{7}{*}{$P=0,321$} \\
\hline \multirow[t]{2}{*}{ Empleado } & Frecuencia & 21 & 9 & 15 & 45 & \\
\hline & Porcentaje & $46,7 \%$ & $20 \%$ & $33,30 \%$ & $100 \%$ & \\
\hline \multirow[t]{2}{*}{ Estudiante } & Frecuencia & 47 & 24 & 21 & 92 & \\
\hline & Porcentaje & $51,1 \%$ & $26,10 \%$ & $22,80 \%$ & $100 \%$ & \\
\hline \multirow[t]{2}{*}{ Total } & Frecuencia & 103 & 46 & 60 & 209 & \\
\hline & Porcentaje & $49,3 \%$ & $22 \%$ & 28,7 & $100 \%$ & \\
\hline
\end{tabular}

Tabla 2. Nivel de Actividad Física de acuerdo al vínculo con la universidad 
Con respecto a los niveles de AF entre sexos, diferencias estad sticamente significativas $(p<0,05)$ se pudieron observar para los niveles alto y bajo, donde, en comparaci n con las mujeres, los hombres reportaron mayor proporci $\mathrm{n}$ de $\mathrm{AF}$ en alta intensidad (56,3\% vs. $43,7 \%)$, mientras que las mujeres reportaron mayores niveles de AF de baja intensidad (65\% vs. $35 \%)$; si bien el nivel de AF moderado fue mayor tambi $n$ para las mujeres $(60,9 \%$ vs. $39,1 \%)$, no se encontraron diferencias estad sticamente significativas ( $p=0,2622)$. Ver Tabla 3.

\begin{tabular}{l|l|c|c|c|c}
\hline \multirow{2}{*}{ Sexo } & \multicolumn{3}{c|}{ Nivel de Actividad Física } & \multirow{2}{*}{ Total } \\
\cline { 3 - 6 } \multicolumn{2}{c|}{} & Alto & Moderado & Bajo & \\
\hline \multirow{2}{*}{ Hombre } & Frecuencia & 58 & 18 & 21 & 97 \\
\cline { 2 - 5 } & Porcentaje & $56,3 \%$ & $39,1 \%$ & $35,0 \%$ & $46,4 \%$ \\
\hline \multirow{2}{*}{ Mujer } & Frecuencia & 45 & 28 & 39 & 112 \\
\cline { 2 - 5 } & Porcentaje & $43,7 \%$ & $60,9 \%$ & $65,0 \%$ & $53,6 \%$ \\
\hline \multirow{2}{*}{ Total } & Frecuencia & 103 & 46 & 60 & 209 \\
\cline { 2 - 5 } & Porcentaje & $49,3 \%$ & $22,0 \%$ & $28,7 \%$ & $100,0 \%$ \\
\hline P & & 0,005 & 0,262 & 0,036 & \\
\hline
\end{tabular}

Tabla 3. Nivel de Actividad Física de acuerdo al sexo

\section{Estados de cambio frente a la Actividad Física}

Los estados de cambio de la AF muestran la siguiente distribuci $n$ en relaci $\mathrm{n}$ al $v$ nculo con la Universidad: la mayor proporci $\mathrm{n}$ la obtuvo el estado de mantenimiento, presentando un 47,2\% para los docentes, $28,9 \%$ para los empleados y $26,1 \%$ para los estudiantes. El estado de acci $n$ se mostr mayor para los empleados con $22,2 \%$, seguido por los estudiantes con $19,6 \%$ y los docentes con $16,7 \%$. Para el estado de preparaci $n$ se identific, al igual que el estado anterior, mayor proporci $\mathrm{n}$ para los empleados con $26,7 \%$, los docentes con $18,1 \%$, y los estudiantes con $13 \%$. Es 
importante resaltar que, solo para los estudiantes, los estados de contemplaci $n$ y precontemplaci $n$ presentaron igual porcentaje $(20,7 \%)$, y fueron los estados de cambio con mayores proporciones despu s del estado de mantenimiento, presentando diferencias estad sticamente significativas entre los diferentes $v$ nculos con la universidad ( $p=0,002)$. Ver Tabla 4.

Los estados de cambio entre sexos presentaron diferencias estad sticamente significativas $(p<0,05)$, para los estados de precontemplaci n (mujeres $75,8 \%$ vs hombres $24,2 \%$ ) y preparaci n (hombres 62,2\% vs. mujeres 37,8\%). Para los estados de contemplaci $n$, acci $n$ y mantenimiento no se encontraron diferencias estad sticamente significativas entre sexos. Ver Tabla 5.

\section{DISCUSIÓN}

El presente estudio permite identificar, de acuerdo con la muestra estudiada, que para el campus universitario de Ciudadela Robledo casi la mitad de las personas presentan niveles de AF altos (estudiantes $51,1 \%$, docentes 48,6\% empleados 46,7\%); los niveles de AF bajos fueron mayores para los docentes y empleados $33,3 \%$ en cada grupo, lo cual se relaciona con la prevalencia de inactividad f sica a nivel mundial, que se ubica en $31,1 \%$ (Hallal et al., 2012), y los resultados de un estudio comparativo desarrollado con profesores de dos universidades en $M$ xico y Espa a, donde se encontr que el 31,3\% de los profesores y 30,6\% de las profesoras de la universidad Mexicana presentan nivel bajo de AF; y para la universidad Espa ola, el 27,3\% de sus docentes y $20 \%$ de sus profesoras (Hall, S enz \& Monreal, 2009).

Si bien no se identificaron diferencias estad sticamente significativas entre los diferentes $v$ nculos con la universidad, se podr a pensar que los niveles $\mathrm{m}$ s elevados encontrados de AF baja en los docentes y empleados es consecuencia de la falta de tiempo para realizar ejercicio $f$ sico en su tiempo libre, debido a sus 


\begin{tabular}{|c|c|c|c|c|c|c|c|c|c|}
\hline \multirow{2}{*}{\multicolumn{2}{|c|}{ 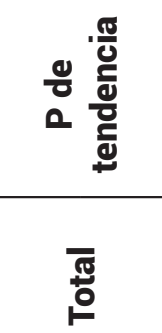 }} & \multicolumn{8}{|c|}{$\begin{array}{l}\text { Na } \\
\text { ○ } \\
\text { O } \\
\text { II }\end{array}$} \\
\hline & & $\mathfrak{N}$ & $\begin{array}{l}\text { ᄋ̊ } \\
\text { ᄋ' }\end{array}$ & $\stackrel{10}{+}$ & $\begin{array}{l}\text { 오 } \\
\text { ᄋ̊ }\end{array}$ & ๙̃ & $\begin{array}{l}\text { ठ̊ } \\
\text { ¿̊ }\end{array}$ & 옹 & $\begin{array}{l}\text { ठ̊ } \\
\text { ¿̊ }\end{array}$ \\
\hline \multirow{5}{*}{ 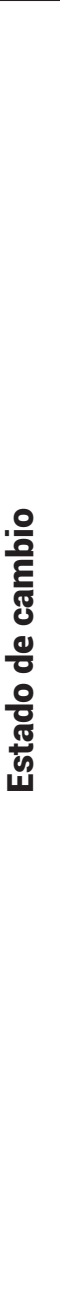 } & 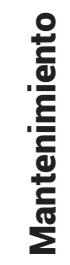 & ஓे & $\underset{\mathrm{N}}{\stackrel{\mathrm{V}}{\mathrm{N}}}$ & $m$ & $\begin{array}{l}\stackrel{\circ}{ } \\
\text { ळे }\end{array}$ & $\underset{\sim}{ \pm}$ & $\frac{0^{\circ}}{\sigma^{-}}$ & $\Gamma$ & $\begin{array}{l}\text { ○े } \\
\text { ले }\end{array}$ \\
\hline & :0웜 & $\stackrel{N}{r}$ & $\begin{array}{l}\stackrel{0}{\wedge} \\
6 \\
0\end{array}$ & $\stackrel{\circ}{\circ}$ & 犬్̊ & $\stackrel{\infty}{-}$ & $\begin{array}{l}\text { 우 } \\
\text { ஸे }\end{array}$ & ㅇ & 윰 \\
\hline & $\begin{array}{l}\text { :응 } \\
\text { 퓷 } \\
\text { 원 }\end{array}$ & $\stackrel{m}{-}$ & $\frac{0^{\circ}}{\infty^{-}}$ & $\stackrel{\sim}{\sim}$ & $\begin{array}{l}\stackrel{\circ}{N} \\
\stackrel{2}{\sigma}\end{array}$ & $\stackrel{\sim}{\sim}$ & $\begin{array}{l}\text { ○े } \\
\text { ஸे }\end{array}$ & $\hat{m}$ & $\begin{array}{l}\stackrel{\circ}{N} \\
\stackrel{-}{-}\end{array}$ \\
\hline & 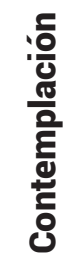 & ما & 今̊ & $\nabla$ & $\begin{array}{l}\stackrel{\circ}{ } \\
\infty\end{array}$ & $\stackrel{\sigma}{\square}$ & $\begin{array}{l}\stackrel{0}{N} \\
\stackrel{ }{\circ}\end{array}$ & $\stackrel{\infty}{\sim}$ & $\begin{array}{l}\stackrel{\circ}{+} \\
\text { ஸे }\end{array}$ \\
\hline & 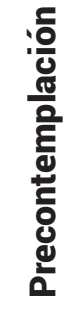 & $\infty$ & $\begin{array}{l}\stackrel{\circ}{\check{E}} \\
\text { Е }\end{array}$ & 6 & $\begin{array}{l}\text { ஹे } \\
\text { m }\end{array}$ & $\stackrel{\sigma}{\sigma}$ & $\begin{array}{l}\stackrel{\circ}{N} \\
\stackrel{ }{N}\end{array}$ & $\stackrel{m}{m}$ & $\begin{array}{l}\text { ○̊ } \\
\infty \\
10 \\
\end{array}$ \\
\hline \multirow{2}{*}{\multicolumn{2}{|c|}{ 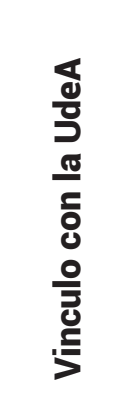 }} & 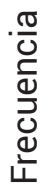 & $\begin{array}{l}\frac{0}{\pi} \\
\stackrel{\pi}{\frac{1}{d}} \\
\frac{0}{0} \\
0\end{array}$ & $\begin{array}{l}\frac{\pi}{U} \\
\frac{C}{U} \\
\frac{U}{U} \\
\frac{d}{L}\end{array}$ & 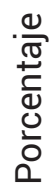 & $\begin{array}{l}\frac{\pi}{U} \\
\frac{C}{U} \\
\frac{U}{U} \\
\frac{d}{L}\end{array}$ & $\begin{array}{l}\frac{0}{\pi} \\
\stackrel{\pi}{\frac{1}{d}} \\
\frac{0}{0} \\
0\end{array}$ & $\begin{array}{l}\frac{\pi}{U} \\
\frac{C}{U} \\
\frac{U}{U} \\
\frac{d}{L}\end{array}$ & $\begin{array}{l}\frac{0}{\pi} \\
\stackrel{\pi}{\frac{1}{d}} \\
\frac{0}{0} \\
0\end{array}$ \\
\hline & & 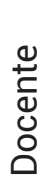 & & $\frac{\frac{O}{0}}{\frac{\pi}{d}}$ & & 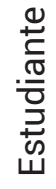 & & $\begin{array}{l}\bar{\pi} \\
\stackrel{0}{0} \\
\end{array}$ & \\
\hline
\end{tabular}




\begin{tabular}{|c|c|c|c|c|c|c|c|c|}
\hline \multicolumn{2}{|c|}{$\begin{array}{l}\bar{\pi} \\
\text { 믕 }\end{array}$} & ô & $\begin{array}{l}\stackrel{\circ}{+} \\
\stackrel{0}{6}\end{array}$ & $\stackrel{N}{\mp}$ & $\begin{array}{l}\text { రొْ } \\
\text { ஸి }\end{array}$ & 오 & $\begin{array}{l}\text { ○े } \\
\text { ○े }\end{array}$ & \\
\hline \multirow{5}{*}{ 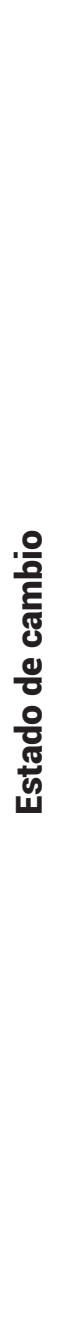 } & 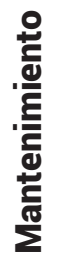 & ి్ల & $\begin{array}{l}\stackrel{\circ}{ } \\
\text { চั }\end{array}$ & & $\frac{\circ 0}{\frac{0}{6}}$ & $\bar{T}$ & $\begin{array}{l}\text { ○े } \\
\text { 犬े }\end{array}$ & 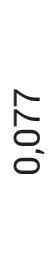 \\
\hline & 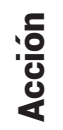 & $\mathfrak{r}$ & $\begin{array}{l}\text { 우 } \\
\stackrel{+}{+} \\
\text { ㅇ }\end{array}$ & $\stackrel{\mathbb{N}}{ }$ & $\begin{array}{l}\text { مْ } \\
\text { مْ } \\
\text { مْ }\end{array}$ & 우 & 우 & $\begin{array}{l}\bar{\infty} \\
\tilde{L} \\
0\end{array}$ \\
\hline & 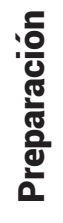 & $\stackrel{\mathbb{N}}{ }$ & $\stackrel{\stackrel{\sim}{N}}{\stackrel{\sigma}{0}}$ & $\Xi$ & $\begin{array}{l}\text { ळे } \\
\text { ஸે }\end{array}$ & $\hat{m}$ & $\frac{\stackrel{\circ}{N}}{N}$ & $\begin{array}{l}\text { गे } \\
\text { ᄋ } \\
\text { ○ }\end{array}$ \\
\hline & 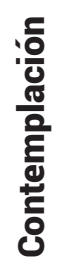 & $\stackrel{\circ}{\circ}$ & $\frac{\stackrel{\circ}{N}}{\sum^{\circ}}$ & $\stackrel{\infty}{-}$ & $\begin{array}{l}\text { ळे } \\
\text { రे }\end{array}$ & $\stackrel{\infty}{\sim}$ & 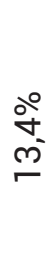 & $\begin{array}{l}\text { V్ } \\
\text { O్ }\end{array}$ \\
\hline & 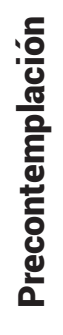 & $\infty$ & $\stackrel{\stackrel{\sim}{+}}{\stackrel{\sim}{\sim}}$ & $\stackrel{\llcorner 0}{\sim}$ & $\begin{array}{l}\text { م0 } \\
\infty \\
0^{0}\end{array}$ & m & 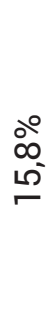 & $\begin{array}{l}\text { L } \\
8 \\
0 \\
0\end{array}$ \\
\hline \multirow{2}{*}{\multicolumn{2}{|c|}{ }} & $\begin{array}{l}\frac{\pi}{0} \\
\frac{\sigma}{d} \\
\frac{d}{0} \\
\frac{d}{4}\end{array}$ & $\begin{array}{l}\frac{0}{\pi} \\
\stackrel{\pi}{ \pm} \\
\frac{0}{0} \\
\frac{0}{0}\end{array}$ & $\begin{array}{l}\frac{\pi}{0} \\
\frac{\sigma}{d} \\
\frac{d}{d} \\
\frac{d}{4}\end{array}$ & 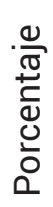 & 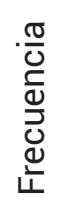 & 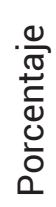 & \\
\hline & & 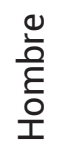 & & \multicolumn{2}{|c|}{$\frac{\overline{\frac{\omega}{2}}}{\frac{2}{\Sigma}}$} & $\begin{array}{l}\bar{\pi} \\
\stackrel{0}{0} \\
\circ\end{array}$ & & 0 \\
\hline
\end{tabular}

Revista Educaci n F sica y Deporte, n. 33(1), 
ocupaciones personales y familiares; sin embargo se encontr , frente a la percepci $n$ de barreras para la pr ctica de la AF relacionadas con los declaraciones: "hacer ejercicio toma mucho de mi tiempo", "hacer ejercicio toma mucho tiempo de las relaciones familiares" $\mathrm{y}$ "hacer ejercicio toma mucho tiempo de mis responsabilidades familiares", que en promedio el $77,3 \%$ de los docentes y el $85,7 \%$ de los empleados no est $n$ de acuerdo con estas afirmaciones (datos no mostrados en este art culo).

Se pudo identificar tambi $n$ que los hombres presentan mayor prevalencia de AF de nivel alto en comparaci $\mathrm{n}$ con las mujeres, $56,3 \%$ y $43,7 \%$ respectivamente; tendencia similar se pudo identificar en el estudio de Varela-Mato et al. realizado en Espa a con estudiantes universitarios, (hombres 38,6\% vs. mujeres 20,9\%) (Varela-Mato, Cancela, Ayan, Mart n \& Molina, 2012).

De manera opuesta, se encontraron mayores proporciones de AF de nivel bajo en las mujeres $65 \%$ y $35 \%$ en los hombres, mostrando diferencias estad sticamente significativas. Si bien no se identificaron diferencias estad sticamente significativas para el nivel de AF moderado entre sexos, se encuentra una diferencia que, para la pr ctica, puede ser importante: las mujeres presentan un $21,8 \% \mathrm{~m}$ s de prevalencia de nivel de AF moderada que los hombres. No obstante, al indagar sobre las barreras relacionadas con las ocupaciones personales y familiares, se encontr frente a las declaraciones: "hacer ejercicio toma mucho de mi tiempo", "hacer ejercicio toma mucho tiempo de las relaciones familiares" $y$ "hacer ejercicio toma mucho tiempo de mis responsabilidades familiares", que tanto los hombres como las mujeres est $\mathrm{n}$ en desacuerdo con estas afirmaciones, hallando un promedio de $74,9 \%$ y $74,7 \%$ respectivamente (datos no mostrados en este art culo), lo cual podr a descartar la falta de tiempo como raz n para no hacer ejercicio, haci ndose necesario plantear estudios que indaguen en detalle las razones por las cuales las mujeres presentan mayor prevalencia de bajo nivel de AF y permitan aclarar esta situaci $n$. 
Frente a los estados de cambio de la AF, se pudo evidenciar que las mujeres presentan mayor proporci $\mathrm{n}$ que los hombres en el estado de precontemplaci $n(75,8 \%$ vs. $24,2 \%)$, lo cual evidencia que estas personas no realizan AF y no tienen la intenci $\mathrm{n}$ de hacerla en los pr ximos seis meses, hallando diferencias estad sticamente significativas $(p<0,05)$. Para el estado de contemplaci $n$, si bien no se encontraron diferencias estad sticamente significativas $(p=0,2226)$, existe una diferencia que, para la pr ctica, podr a ser representativa: las mujeres mostraron un $28,6 \% \mathrm{~m}$ s de prevalencia en este estado frente a los hombres (64,3\% vs. $35,7 \%)$. Resultados similares se evidenciaron en el estudio de Olivares et al. realizado con estudiantes universitarios en Chile, en el cual se encontr que las mujeres presentan mayores proporciones que los hombres para los estados de precontemplaci n y contemplaci n (Olivares, Lera \& Bustos 2008). El estado de preparaci $n$ fue el otro estado en el que se identificaron diferencias estad sticamente significativas $(p<0,05)$, evidenciando mayor proporci $n$ para los hombres, en comparaci $n$ con las mujeres $(62,2 \%$ vs 37,8$)$, datos que se relacionan de manera opuesta con los encontrados en el estudio de Pinto et al., desarrollado con estudiantes universitarios brasile os, en el cual las mujeres presentaron mayor proporci n que los hombres (39,6\% vs. 22,8\%), ( $p<0,05)$. (Pinto, dos Santos, \& Correa, 2006).

Se pudo determinar tambi $n$, con relaci $n$ al v nculo con la universidad, que el estado de cambio que present mayores proporciones fue el de mantenimiento, lo que significa que estas personas realizan AF desde hace $\mathrm{m}$ s de seis meses (docentes $47,2 \%$, empleados $28,9 \%$ y estudiantes $26,1 \%$ ); datos similares se hallaron en el estudio de Giraldo et al. desarrollado con personal docente y administrativo de una universidad Colombiana, en el cual $43,4 \%$ de docentes y $31,2 \%$ de empleados se encontraban en este estado de cambio (Giraldo, Zapata, \& Granada, 2009). 


\section{Limitaciones}

El estudio tuvo una frecuencia de respuesta global del 35,3\%, debido a las dificultades para la ubicaci $\mathrm{n}$ de las personas que fueron seleccionadas, a quienes se trat de contactar empleando diversas estrategias. Otra limitaci n conocida fue la falta de validaci $\mathrm{n}$ en Colombia del instrumento

\section{CONCLUSIONES}

Es importante resaltar que, con base en la muestra estudiada, la mitad de los estudiantes del campus de Ciudadela Robledo tiene niveles altos de $\operatorname{AF}(51,1 \%)$, y casi la mitad de los docentes y empleados alcanzan esta proporci $n(48,6 \%$ y $46,7 \%$ respectivamente). Sin embargo, tanto los docentes como los empleados presentan un $33,3 \%$ de baja $\mathrm{AF}$, lo cual se relaciona con los niveles mundiales de inactividad $\mathrm{f}$ sica $(31,1 \%)$. Al analizar los niveles de AF por sexo, se pudo identificar que los hombres muestran mayor nivel de AF alta en relaci $\mathrm{n}$ con las mujeres (56,3\% y $43,7 \%$ respectivamente). Los niveles bajos de AF tuvieron mayor prevalencia en las mujeres (65\%), en comparaci $n$ con los hombres (35\%). Teniendo en cuenta los resultados encontrados, se hace necesario desarrollar futuras investigaciones en esta poblaci $n$, que permitan develar las causas por las cuales se encuentran diferencias tan acentuadas en los niveles de AF entre sexos, posibilitando una mejor comprensi $\mathrm{n}$ de este fen meno y al mismo tiempo que puedan contribuir con la generaci $n$ de estrategias para incrementar los niveles de AF en esta poblaci n, en especial en las mujeres.

Vale la pena resaltar las proporciones encontradas para el estado de preparaci $n$, el cual hace referencia a aquellas personas que no realizan $A F$ en el tiempo libre, pero tienen la intenci $n$ de hacerla en los pr ximos $30 \mathrm{~d}$ as $(62,2 \%$ para las mujeres 
y $37,8 \%$ para los hombres), evidenci ndose nuevamente un mayor porcentaje en las mujeres. Ser a importante adelantar estrategias que permitan llevar a estas personas al estado de acci n, pues representan una cantidad no despreciable de hombres y en especial de mujeres, que podr an ser motivadas para dar inicio a un programa regular de AF en el tiempo libre.

Con los resultados del presente estudio se espera que las directivas encargadas de liderar los programas deportivos y de AF en el campus universitario de Ciudadela Robledo, tengan algunos insumos que les facilite crear estrategias para mejorar los programas existentes o generar nuevos programas, que busquen incrementar los niveles de AF de la poblaci $\mathrm{n}$ del campus y garanticen una adecuada adherencia a stos, as como la contribuci $\mathrm{n}$ en la adopci $\mathrm{n}$ de $\mathrm{h}$ bitos de vida saludables que incluyan la AF como parte fundamental.

\section{REFERENCIAS}

1. Abu-Omar, K., \& R tten, A. (2008). Relation of leisure time, occupational, domestic, and commuting physical activity to health indicators in Europe. Preventive Medicine, 47(3), 319-323.

2. Aguilar, E. E. R., Zapata, M. H. L., Giraldo, F. J. L., Tejada, J. L. C., \& Vidales, S. A. Z. (2008). An lisis descriptivo de las variables: nivel de actividad $\mathrm{f}$ sica, depresi $\mathrm{n}$ y riesgos cardiovasculares en empleados y docentes de una instituci $\mathrm{n}$ universitaria en Medell $\mathrm{n}$ (Colombia). Apunts. Medicina de l'Esport, 43(158), 55-61.

3. Bauman, A. E., Nelson, D. E., Pratt, M., Matsudo, V., \& Schoeppe, S. (2006). Dissemination of Physical Activity Evidence, Programs, Policies and Surveillance in the International Public Health Arena. American Journal of Preventive Medicine, 31(4, Supplement 1), 57-65.

4. Bull, F. C., Maslin, T. S., \& Armstrong, T. (2009). Global physical activity questionnaire (GPAQ): nine country reliability and validity study. J Phys Act Health, 6(6), 790-804.

5. DANE Departamento Administativo Nacional de Estad stica (sf). Estratificaci n Socioecon mica. Recuperado el 11-06-2013 de: https:// www.dane.gov.co/index.php?option=com_content\&view=article\&id= 354\&Itemid $=114$ 
6. Farinola, M. (2011). Nivel de actividad $\mathrm{f}$ sica en estudiantes universitarios con especial referencia a estudiantes de profesorado en educaci $n$ f sica. Revista Electr nica de Ciencias Aplicadas al Deporte, 4(12).

7. Giraldo, J. C., Zapata, C. D., \& Granada, P. (2009). Caracterizaci n de imaginarios de los empleados de la UTP con relaci $n$ a la pr ctica de la actividad f sica: 2008. Investigaciones Andina, 11(19), 81-92.

8. Hall, J. A., S enz-L pez, P., \& Monreal, L. R. (2009). Comparative study of the physical activity level, nutrition state and abdominal obesity in physical education professors from the University Autonomus of Sinaloa and Huelva University. Retos: Nuevas Perspectivas de Educaci $n$ F sica, Deporte y Recreaci n, 15, 5-8.

9. Hallal, P. C., Andersen, L. B., Bull, F. C., Guthold, R., Haskell, W., \& Ekelund, U. (2012). Global physical activity levels: surveillance progress, pitfalls, and prospects. The Lancet, 380(9838), 247-257.

10. ICBF Instituto Colombiano de Bienestar Familiar, MPS Ministerio de la Protecci n Social, INS Instituto Nacional de Salud, \& Profamilia (2011). Encuesta Nacional de la Situaci n Nutricional en Colombia. Colombia: Los autores.

11. Olivares, S., Lera, L., \& Bustos, N. (2008). Etapas del cambio, beneficios y barreras en actividad $f$ sica y consumo de frutas y verduras en estudiantes universitarios de Santiago de Chile. Rev Chil Nutr, 35(1), 25-35.

12. OMS Organizaci n Mundial de la Salud (2007). El m todo STEPwise de la OMS para la vigilancia de los factores de riesgo de las enfermedades $\mathrm{Cr}$ nicas. Ginebra: La Organizaci n.

13. OMS Organizaci n Mundial de la Salud (2010). Recomendaciones mundiales sobre actividad $f$ sica para la salud. Ginebra: La Organizaci n.

14. Pe a, E., Gallo, E., \& V squez, A. (2009). Actividad f sica en empleados de la Universidad de Caldas, Colombia. Hacia la Promoci $n$ de la Salud, 14(2), 52-65.

15. Pinto Guedes, D., dos Santos, C. A., \& Correa Lopes, C. (2006). Stages of behavior change and habitual physical activity in college students. Revista Brasileira de Cineantropometria e Desempenho Humano, 8(4), 5-15.

16. SSM Secretar a de Salud de Medell $n$ (2008). Diagn stico del riesgo cardiovascular global, evaluaci $n$ de su impacto poblacional, Medell $n$ y sus corregimientos 2007-2008. Medell n: La Secretar a.

17. UdeA Universidad de Antioquia (1996). Acuerdo Superior 083 de 1996. Medell n: La Universidad.

18. UdeA Universidad de Antioquia. (2005). Resoluci n Rectoral 20535 del 17 de marzo de 2005. Medell n: La Universidad.

19. US Department of Health and Human Services. (2008). Physical Activity Guidelines for Americans. Be active, healthy and happy. USA: The Department. 
20. Varela-Mato, V., Cancela, J., Ayan, C., Mart n, V., \& Molina, A. (2012). Lifestyle and Health among Spanish University Students: Differences by Gender and Academic Discipline. International Journal of Environmental Research and Public Health, 9(8), 2728-2741.

Recepción: 05-05-2013

Aprobación: 05-03-2014 